NACIONAL

\title{
El derecho de participación de niños, niñas $y$ adolescentes desde el derecho internacional de los derechos humanos, y su aproximación al proyecto de ley de identidad de género en Chile
}

\author{
The right of participation of children and adolescents from the international law \\ of human rights, and its approximation to the gender identity law draft in Chile
}

\section{Lavinia Francesconi}

Universidad de Leiden, Países Bajos

\begin{abstract}
RESUMEN El presente artículo pretende hacer una breve recopilación de los principales estándares internacionales de derechos humanos relativos al derecho a la participación de niños, niñas y adolescentes. Se analizan someramente los principales referentes normativos en el sistema universal de protección de los derechos humanos y en el Sistema Interamericano de Derechos Humanos en esta materia. El artículo termina con algunas reflexiones sobre el nuevo proyecto de ley de identidad de género que desde 2013 se discute en el Parlamento chileno. El texto tratará de dar a entender si dichos estándares preexistentes han sido incorporados por parte del legislador chileno, con el fin último de aportar a esta discusión la perspectiva del derecho internacional de los derechos humanos. Indudablemente, la participación es solamente uno de los numerosos derechos que se ven involucrados en este debate; aun así, el estudio de la participación desde la rama del derecho internacional de los derechos humanos otorga otra mirada a favor de la inclusión de niños, niñas y adolescentes en el proyecto mencionado.
\end{abstract}

PALABRAS CLAVE Participación, estándares internacionales, derecho internacional de los derechos humanos, niños, niñas y adolescentes, proyecto de ley.

ABSTRACT This article aims to show a selection of the main international human rights legal standards regarding children's right of participation. It analyses briefly the principal legal frame both of the universal human rights protection system and of the Inter-American Human Rights System in the field of participatory rights of children. The essay ends with some reflections on the new Chilean draft law on gender identity, which has been discussed since 2013 in the Chilean Parliament. The article analyzes if the pre-existing international standards have been incorporated by the Chilean law- 
maker, with the ultimate purpose of contributing to the current debate within the perspective of the International Human Rights Law. Of course, participation is just one of the numerous rights which are challenged in this debate, but the study of this particular right from the international human rights point of view ensures an alternative look on behalf of the inclusion of children within the mentioned draft law.

KEYWORDS Participation, international standards, human rights law, children, gender identity, draft legislation.

\section{Introducción}

Los «estándares internacionales» pueden entenderse, de forma general, como estándares jurídicos, elaborados en sede internacional, esto es, por parte de organizaciones internacionales. Este corpus iuris normalmente define las obligaciones mínimas que debiesen ser respetadas en una determinada materia. En otras palabras, éstos son indicadores que detallan el contenido de una norma, lo cual implica que los Estados que reconozcan un determinado derecho ${ }^{1}$ tendrán la obligación de respetarlo, protegerlo, garantizarlo y promoverlo a través de medidas que deben ser en algunos casos positivas y en otras negativas (Serrano, 2013).

Ahora bien, aunque se considere que los tratados y la costumbre constituyen las principales fuentes legales en del derecho internacional público, y por ende también del derecho internacional de los derechos humanos, otros tipos de fuentes que se adopten en el ámbito internacional - como declaraciones, resoluciones, directrices, etcétera- son cruciales para su interpretación y aplicación. Lo anterior se entiende considerando que el texto de los tratados define normalmente un umbral mínimo de protección, cuyo alcance y contenido especifico es posible entender a través de instrumentos complementarios, sin dejar de lado que la conformación del derecho internacional público históricamente se ha basado en la consolidación de prácticas que estos documentos consagran.

Es así como se vuelve interesante el ejercicio de entender si en un determinado contexto nacional se responde a estas obligaciones por parte del ordenamiento interno, a través de la integración de las directrices de los estándares. ${ }^{2}$

En las líneas que siguen, se esbozará cuáles son los denominados estándares del derecho internacional de los derechos humanos sobre el derecho de participación

1. Teniendo siempre presente que algunos derechos son de aplicación universal, independientemente de su reconocimiento formal, establecidos por normas consuetudinarias. Me refiero a las normas de jus cogens.

2. Según Burgorgue-Larsen (2013: 15-30), la adhesión al estándar dependerá del grado de internacionalización de las respectivas constituciones, como también del grado de persuasión de la fuente internacional. 
de niños, niñas y adolescentes, y si el legislador chileno ha incorporado este marco normativo preexistente en el debate en torno al proyecto de ley sobre identidad de género. Por ende, el objetivo de investigación se traduce en el análisis del proyecto a la luz del derecho internacional de los derechos humanos solo respecto de un derecho específico de determinados sujetos jurídicos, por lo que deja de lado la teorización sobre la identidad de género y el estudio de otros aspectos del proyecto.

\section{Estándares internacionales de derechos humanos sobre participación de niños, niñas y adolescentes}

Respecto de los derechos de niños, niñas y adolescentes, el instrumento principal es, sin duda, la Convención sobre los Derechos del Niño. Adoptada en 1989 y ratificada por Chile en 1990, esta convención marcó un cambio de paradigma en cuanto al entendimiento y percepción de este grupo (Bartos, 2016: 15-30), especialmente en su subjetividad como titulares de derechos, lo que incluye derechos de participación (Holzscheiter, 2010: 153). Su gran número de ratificaciones da cuenta de la existencia un fuerte índice de consenso entre los Estados sobre el contenido y la aplicación universal de los derechos de niños, niñas y adolescentes (Holzscheiter, 2010: 145).

Aunque los derechos de la Convención son indivisibles y están interconectados, ${ }^{3}$ los cuatros principios generales, que deben guiar su observancia e interpretación de forma transversal, son el derecho a la no discriminación (artículo 2), la consideración primordial del interés superior del niño (artículo 3 ), el derecho a la vida y el desarrollo (artículo 6) y el derecho del niño a expresar su opinión libremente en todos los asuntos que lo afectan, y que dicha opinión sea debidamente tomada en cuenta (artículo 12) (Kilkelly, 2011: 179).

Desde 1989, la interpretación de este artículo ha ido evolucionando, entendiéndose hoy día no solamente como el derecho del niño, niña o adolescente a ser oído, sino que de forma más general como participación, es decir:

Procesos permanentes, como intercambios de información y diálogos entre niños y adultos sobre la base del respeto mutuo, en que los niños puedan aprender la manera en que sus opiniones y las de los adultos se tienen en cuenta y determinan el resultado de esos procesos. ${ }^{4}$

Es así como este artículo ha enfatizado la importancia y la diferencia de la voz de niños, niñas y adolescentes respecto de la de los adultos, lo que cambió el modelo clásico que los entendía como objeto de protección, hacia la concepción como parti-

3. Comité de los Derechos del Niño, Observación General 5, 27 de noviembre de 2003, CRC/ $\mathrm{GC} / 2003 / 5$, folio 5 .

4. Comité de los Derechos del Niño, Observación General 12, 20 de julio de 2009, CRC/C/GC/12. 
cipantes activos en sus propias vidas (Bartos, 2016: 155-116). ${ }^{5}$ Los dos párrafos del artículo 12 no otorgan a niños, niñas y adolescentes el derecho a la autodeterminación, pero sí el derecho a participar en la toma de decisiones, apuntando a su condición jurídica y social, la cual, por un lado, carece de la plena autonomía que tienen los adultos, pero por el otro, sigue siendo sujeto de derechos (Bartos, 2016: 1). ${ }^{6}$

$\mathrm{El}$ artículo 12 no es el único pertinente en la materia. De hecho, debe considerarse en conjunto con el artículo 13 (libertad de expresión), ${ }^{7}$ el artículo 14 (libertad de pensamiento, conciencia y religión) (Unicef, 2007: 186) y el artículo 15 (libertad de asociación) (Naciones Unidas, 1997: 437). ${ }^{8}$ La contemplación de los artículos mencionados en su conjunto deja en claro que el «niño es una persona detentora [o titular] de derechos humanos fundamentales, opiniones y sentimientos propios» (Unicef, 2007: 149).

Ahora bien, a fin de entender el alcance del derecho de participación, asumen un rol primordial las observaciones y recomendaciones generales emanadas del Comité de los Derechos del Niño, órgano encargado de vigilar el respeto y la protección de los derechos establecidos en el tratado internacional que lo crea. Las observaciones permiten hacer una interpretación evolutiva del texto del tratado, lo que cumple con la función de aclarar el contenido de sus disposiciones y definir el contenido de las obligaciones contraídas por los Estados partes. ${ }^{9}$ Es así como, para mejor explicar el alcance del artículo 12, es menester referirse a la observación general sobre él emanada por el Comité de los Derechos del Niño.

5. Véase también Comité de los Derechos del Niño, Observación General 12.

6. El texto del artículo 12 reza: «Los Estados partes garantizarán al niño que esté en condiciones de formarse un juicio propio el derecho de expresar su opinión libremente en todos los asuntos que afectan al niño, teniéndose debidamente en cuenta las opiniones del niño, en función de la edad y madurez del niño. Con tal fin, se dará en particular al niño oportunidad de ser escuchado en todo procedimiento judicial o administrativo que afecte al niño, ya sea directamente o por medio de un representante o de un órgano apropiado, en consonancia con las normas de procedimiento de la ley nacional».

7. Aunque los artículos 12 y 13 aparentemente tienen que ver con el mismo ámbito: «While Article 13 recognizes in a general way freedom of expression, Article 12 should prevail in all those cases where the matters at stake affect the child, while stressing the right of the child to be heard and for the child's views to be taken into account» («Mientras el artículo 13 reconoce, en general, la libertad de expresión, el artículo 12 debe prevalecer en todos los casos donde los asuntos en cuestión afecten a los niños, en tanto se enfatiza el derecho del niño a ser oído y para que se tengan en cuenta sus opiniones» (Naciones Unidas, 1997: 427).

8. Este artículo reconoce la libertad de asociación y de reunión pacífica, las cuales son un reflejo de los derechos participatorios estatuidos en el artículo 12 de la Convención.

9. En cuanto a la fuerza jurídica de las observaciones, y para una revisión de las diferentes posiciones sobre el reconocimiento de las recomendaciones como «interpretación autoritativa»o como práctica del tratado exartículo 31.3.c de la Convención de Viena de 1969 sobre los Tratados, véase Keller y Grover (2012: 129 y ss.). 
En cuanto a las «condiciones de formarse un juicio propio», según el Comité los Estados no deben actuar en la creencia de que los niños, niñas y adolescentes no estén capacitados para formar su opinión, sino que, al revés, deberán actuar presumiendo que tengan esa capacidad, dado que no corresponde al niño probar lo contrario. ${ }^{10}$ Además, no deberá imponerse límite de edad para la expresión de dicha opinión. De hecho, la propia Convención no lo establece, justamente a raíz de la constatación de que los niños, niñas y adolescentes pueden formar su opinión desde pequeños, aunque no sean capaces de comunicarla. En este sentido, adquieren relevancia otras formas de expresión y comunicación. ${ }^{11}$

Cuando se lee «el derecho de expresar su opinión libremente», hay que reconocer el adverbio «libremente» bajo el entendido de que los niños, niñas y adolescentes deben participar sin ninguna presión externa, sin manipulaciones o influencias indebidas. También implica que pueden escoger si quieren o no ejercer sus derechos. De acuerdo con lo anterior, se exige que los responsables de escucharlos y los padres o tutores informen al niño de los asuntos, las opciones y las posibles decisiones que pueden adoptarse y sus consecuencias; esto es, el ejercicio del derecho a ser informado.

Por otro lado, la segunda condición que se exige para la aplicación de este derecho es que los niños, niñas y adolescentes debe ser escuchados «en todos los asuntos que afectan al niño». No existe un listado de estas situaciones, dado que prácticamente podrían considerarse todo tipo de instancias que de una forma o de otra pueden afectarlos. ${ }^{12}$

Sucesivamente, el artículo enuncia: «Teniéndose debidamente en cuenta las opiniones del niño, en función de la edad y madurez del niño». ${ }^{13}$ Se debe tomar «debidamente en cuenta» la opinión de niños, niñas y adolescentes, porque no basta con

10. Para determinar la capacidad de niños, niñas y adolescentes, el Comité sugiere como medida la de tomar en cuenta la opinión a partir de un análisis caso por caso, para determinar si ellos son o no capaces de formarse un juicio propio de manera razonable e independiente. Entonces, en el análisis que se desarrolle, el respectivo encargado de adoptar decisiones debe considerar las opiniones expresadas por niños y adolescentes «como un factor destacado en la resolución de la cuestión» (Observación General 12, párrafo 44).

11. «En tal sentido, las formas de comunicación no verbal toman mayor relevancia más allá de la edad: el juego, el lenguaje corporal, expresiones faciales, el dibujo y la pintura son los principales medios para demostrar la comprensión, las opciones y preferencias» (IIN, 2011: 15).

12. Observación General 12, páginas 26-27. En este sentido, es interesante señalar, a modo de ejemplo, cómo la contaminación del medioambiente también es un asunto que afecta a niños, niñas y adolescentes, como muestra el caso Juliana y otros con Estados Unidos de 2015, en el que un grupo de niños y adolescentes demandó al Gobierno Federal de Estados Unidos por no haber contrarrestado la emisión de gases fósiles, lo que contribuyó negativamente al cambio climático. La información del caso se encuentra disponible en http://bit.ly/2IMJuPM.

13. Observación General 12, páginas 20-21. 
escucharlos en forma activa, sino que deben ser efectivamente entendidos y valorados. Por otro lado, cuando se habla de madurez y edad, se quiere dejar en claro que la edad per se no puede determinar la trascendencia de estas opiniones, y debe verse caso a caso. Mientras más grandes sean los efectos del resultado en la vida del niño, más importante será la correcta evaluación de la madurez de éste, de acuerdo con el concepto de la evolución de las facultades del niño, según el dictamen del artículo $5,{ }^{14}$ lo cual a su vez implica que, a medida que van adquiriendo competencias cada vez mayores, se reduce su necesidad de orientación y aumenta su capacidad de asumir responsabilidades sobre las decisiones que afectan sus vidas. «De este modo, a la madurez se le interpreta como una capacidad de respuesta razonable e independiente ante situaciones, en la que demuestran procesos de comprensión y evaluación de los hechos particulares» (IIN, 2011: 16).

Ahora bien, ya que el análisis parte de los denominados «estándares internacionales», no es baladí mencionar que el referente en ámbito interamericano en materia se puede encontrar en la Opinión Consultiva emitida por la Corte Interamericana de Derechos Humanos sobre la condición jurídica y derechos humanos del niño (2002). Debido a la naturaleza de la consulta, la Corte no ahonda en la temática de la participación de niños, niñas y adolescentes más allá de la participación en procesos administrativos y judiciales, pues se remite a la Convención y a la jurisprudencia del Comité de los Derechos del Niños y del Comité de Derechos Humanos, por lo que explicita que se debe procurar el mayor acceso del menor en esos casos. ${ }^{15}$

Existe también un desarrollo jurisprudencial por parte de la Corte en relación con niños, niñas y adolescentes en general, y también sobre el derecho a participar, que desarrolla de nuevo a instancias de la Convención de los Derechos del Niño y de la Observación 12 del Comité, que se relaciona con los artículos 8 y 25 de la Convención Americana de Derechos Humanos. ${ }^{16}$ La Corte configura así los derechos al desarrollo progresivo de los derechos de los niños y adolescentes, sobre la base de un examen caso a caso, y según su derecho a ser informado; a que sus opiniones sean tomadas en cuenta y, de lo contrario, que la autoridad respectiva argumente por qué no considera

14. Observación General 12, páginas 28-31. Entendiendo que «el concepto de facultades en evolución ocupa un lugar central entre [...] el reconocimiento de los niños como protagonistas activos de su propia vida, con la prerrogativa de ser escuchados y respetados y de que se les conceda una autonomía cada vez mayor en el ejercicio de sus derechos, y la necesidad que tienen, al mismo tiempo, de recibir protección en función de su relativa inmadurez y corta edad» (Lansdown, 2005: 19).

15. Opinión Consultiva OC-17/2002, Corte Interamericana de Derechos Humanos, 28 de agosto de 2002, «Condición jurídica y derechos humanos del niño», párrafos 101-102.

16. De hecho, la misma Corte estima que la Convención de los Derechos del Niños y la Convención Americana de Derechos Humanos «forman parte de un muy comprensivo corpus juris internacional de protección de los niños». Véase la sentencia del caso «Niños de la Calle» (Villagrán Morales y otros) con Guatemala, Corte Interamericana de Derechos Humanos, fondo, 19 de noviembre 1999, párrafo 194. 
la opinión de los niños, niñas y adolescentes; ${ }^{17}$ que puedan participar de forma significativa también en procedimientos como el de $\operatorname{asilo}^{18} \mathrm{y}$ de la mayor forma posible, refiriéndose en este caso a niños, niñas y adolescentes con discapacidad. ${ }^{19}$

\section{Consideraciones en torno al proyecto de ley de identidad de género a raíz del derecho internacional de los derechos humanos}

El proyecto de ley sobre identidad de género ingresó en 2013, planteado por la preocupación nacional sobre la identidad de género y la regularización de la situación de las personas «cuyo nombre y sexo registrado es incongruente con su identidad de género». ${ }^{20}$

En concreto, se habla de la rectificación del nombre y sexo registral de la partida de nacimiento. El debate se ha concentrado en los requisitos para acceder a este trámite - entre otros, la disposición de informes de salud y psiquiátricos-, el procedimiento per se (administrativo o judicial), que tiene en cuenta si la persona involucrada había contraído vínculo matrimonial, la inclusión de niños, niñas y adolescentes y las modalidades de dicha inclusión según un rango etario. ${ }^{21}$ Después de cinco años, se han elaborado dos proyectos por parte del Senado y la Cámara de Diputados, en los cuales se encuentran diferencias notables. Por falta de aprobación en su tercer trámite constitucional, el proyecto está siendo actualmente analizado por una comisión mixta que tiene por objetivo despachar el proyecto a la brevedad. ${ }^{22}$

Considerando el objeto de investigación del presente artículo, el siguiente análisis no se centra en la materia que el proyecto pretende regular, esto es, la «identidad de género», ${ }^{23}$ sino en la regulación de la inclusión de niños, niñas y adolescentes en las

17. Sentencia del caso Atala Riffo y Niñas con Chile, Corte Interamericana de Derechos Humanos, serie c 239, 24 de febrero de 2012, párrafos 196-200.

18. Sentencia del caso Familia Pacheco Tineo con Bolivia, Corte Interamericana de Derechos Humanos, serie c 272, 25 de noviembre de 2013, párrafo 223.

19. Sentencia del caso Furlan y Familiares con Argentina, Corte Interamericana de Derechos Humanos, serie c 246, 31 de agosto de 2012, párrafo 230.

20. Moción parlamentaria del 7 de mayo de 2013, disponible en http://bit.ly/2C5Qm9N.

21. Para un estudio exhaustivo de la evolución del texto legislativo y de su debate, véase Meza-Lopehandía (2018).

22. Los hitos de la tramitación pueden consultarse en el sitio de la Cámara de Diputados de Chile, disponible en http://bit.ly/2 55 Qm9N.

23. Que desde el derecho internacional de los derechos humanos se define como «la vivencia interna e individual del género tal como cada persona la siente profundamente, la cual podría corresponder o no con el sexo asignado al momento del nacimiento, incluyendo la vivencia personal del cuerpo (que podría involucrar la modificación de la apariencia o la función corporal a través de medios médicos, quirúrgicos o de otra índole, siempre que la misma sea libremente escogida) y otras expresiones de género, incluyendo la vestimenta, el modo de hablar y los modales» (Principio 19 de Yogyakarta, sobre «Derecho a la libertad de opinión y expresión»). 
dos versiones del proyecto de ley actualmente disponibles, y en específico su «participación protagónica», que debiese permitirle ejercer un «rol central» en su evolución, lo cual desafía las relaciones adultocéntricas según las costumbres socioculturales (Mundaca Rojas y Flores Zúñiga, 2014: 128).

Es menester mencionar que, en la primera versión del proyecto aprobada por el Senado, su artículo 5 establece la minoría de edad como causal de inadmisibilidad de la solicitud de cambio de sexo registral: «El o la oficial del Servicio de Registro Civil e Identificación declarará inadmisible la solicitud cuando quien la formulare fuere menor de edad». ${ }^{24}$ Sin entrar en el detalle, el no hacerse cargo de la situación de los menores de edad en cuanto a su identidad de género implicaba ignorar rotundamente cada uno de los principios rectores de la Convención de los Derechos del Niño, y por ende las obligaciones internacionales en la materia especificadas por los enunciados del Comité de los Derechos del Niño, según los cuales se entiende que ellos debiesen ser incluidos en los procedimientos contemplados en el proyecto de ley, pues el tema de su identidad es claramente «un asunto que los afecta», inclusive según el artículo 8 de la Convención, que explicita la obligación de los Estados a respetar la identidad de los niños, niñas y adolescentes. De hecho, el adverbio «libremente» que se lee en el artículo 12 también significa «sin limitaciones», es decir, que no existe un sector reservado únicamente a la autoridad de los adultos donde no tenga cabida la opinión de los niños. ${ }^{25}$ Es más, el mismo artículo empieza con el verbo «garantizarán», lo cual, en palabras del Comité: «Es un término jurídico de especial firmeza, que no deja margen a la discreción de los Estados partes» y que implica dos tipos de obligaciones: «Que existan mecanismos para recabar las opiniones del niño sobre todos los asuntos que lo afectan y tener debidamente en cuenta esas opiniones». ${ }^{26}$

Por otro lado, el proyecto de la Cámara eliminó este requisito, lo que permitiría la inclusión de menores de edad; sin embargo, no creó un procedimiento específico para que puedan acceder al cambio de sexo registral. ${ }^{27}$ Lo anterior no es menor, si consideramos la importancia que la participación de niños, niñas y adolescentes «no debe tratarse de cualquier tipo de participación, sino de una participación significativa y protagónica» (CIDH, 2017: 310) Se puede de todos modos deducir que la situación de niños, niñas y adolescentes es equiparada a la de las personas con vínculo matrimonial. Esta equiparación puede concluirse a partir de diversos artículos que hacen referencia a ambas categorías. Por ejemplo, el artículo 10 del proyecto se refiere al «procedimiento iniciado en el caso de la solicitud efectuada por una persona sujeta a vínculo matrimonio no disuelto o por un niño, niña o adolescente» y establece

24. Oficio 118/SEC/17, 14 de junio de 2017, Senado de Chile, disponible en http://bit.ly/2C5Qm9N.

25. Observación General 12, páginas 22-25.

26. Observación General 12, página 19.

27. Oficio 13.738, 23 de enero de 2018, Cámara de Diputados, disponible en http://bit.ly/2C5Qm9N. 
que «el tribunal ordenará en la misma sentencia [...] la rectificación de la partida de nacimiento». Una referencia parecida se hace en el artículo 11, relativa a la emisión de nuevos documentos una vez aceptada la solicitud de rectificación o dictada la respectiva sentencia.

El debate legislativo se ha agudizado en torno a la participación de niños, niñas y adolescentes y de su modalidad, mientras que el Ejecutivo ha explicitado su postura en las siguientes indicaciones: solo los mayores de catorce años y menores de dieciocho años podrán acceder al cambio registral; en los casos en que se permita, incluso con acuerdo de la familia del menor, el cambio registral será posible solo mediante resolución judicial, por lo que deberá seguirse un procedimiento judicial ante el Tribunal de Familia competente.

Ahora bien, a la luz de los estándares de derecho internacional de los derechos humanos descritos en un comienzo, y en cuanto a las limitaciones etarias sugeridas y mantenidas en las últimas indicaciones del Gobierno, éstas podrían resultar contrarias a recomendaciones elaboradas por parte de la doctrina a nivel nacional (Espejo Yaksic y Lathrop Gómez, 2015: 412), así como respecto de lo enunciado anteriormente sobre la capacidad de formarse la propia opinión (artículo 12 de la Convención de los Derechos del Niño) que, según lo establecido por el Comité de los Derechos del Niño, debería ser evaluada caso por caso, sin que se deban imponer límites de edad para que los niños, niñas y adolescentes puedan expresar su opinión.

Si consideramos que la identidad de género forma parte de los asuntos que afectan a niños y adolescentes según las diferencias que existen en el desarrollo de cada etapa de crecimiento, el Comité insta a los Estados a escuchar sus opiniones siempre que manifiesten interés por intervenir. ${ }^{28}$ Por otro lado, esta opinión debería ser «debidamente tomada en cuenta» para que los niños, niñas y adolescentes puedan tomar decisiones en torno a su vida y su desarrollo como sujetos de derecho.

La autonomía progresiva es otra norma que va reforzando lo anterior, ${ }^{29}$ según la cual los niños, niñas y adolescentes deberían ejercer sus derechos de acuerdo con sus proyectos de vida y siempre en la medida de sus capacidades para poner en práctica sus derechos, esto es, de acuerdo con su desarrollo como persona. Dicho en otras palabras, a medida que los niños adquieren facultades, tienen derecho a asumir un nivel cada vez mayor de responsabilidad respecto de la regulación de los asuntos

28. Observación General 12, páginas 26-27.

29. El artículo 5 de la Convención garantiza a los niños, niñas y adolescentes el ejercicio personal de sus derechos, para que puedan desempeñar sus prerrogativas: «Los Estados partes respetarán las responsabilidades, los derechos y los deberes de los padres o, en su caso, de los miembros de la familia ampliada o de la comunidad, según establezca la costumbre local, de los tutores u otras personas encargadas legalmente del niño de impartirle, en consonancia con la evolución de sus facultades, dirección y orientación apropiadas para que el niño ejerza los derechos reconocidos en la presente Convención». 
que los afectan..$^{30} \mathrm{La}$ evolución de esas facultades tiene que ver con los «procesos de maduración y de aprendizaje por medio de los cuales los niños adquieren progresivamente conocimientos, competencias y comprensión, en particular comprensión de sus derechos, y sobre cómo dichos derechos pueden materializarse mejor». ${ }^{31}$

\section{Conclusiones}

El derecho a la participación permite hacer interesantes análisis en relación con los derechos de niños, niñas y adolescentes, lo cual es también uno de los pilares fundantes de la Convención de los Derechos de Niño. Hemos visto que el derecho internacional de los derechos humanos determina el umbral mínimo de protección de ciertos derechos, que normalmente viene, por decir, complementados por los dictámenes de órganos internacionales de derechos humanos.

En el caso de estudio, estas normas jurídicas internacionales adquieren relevancia y pertinencia frente a la controversia legislativa sobre el proyecto de ley de identidad de género, por su ámbito de aplicación. Sin ir más allá del derecho a la participación strictu sensu, examinado a partir del derecho internacional de los derechos humanos, puede deducirse que, por un lado, la inclusión de niños, niñas y adolescentes en estos procedimientos se justifica por el hecho de que la identidad, en todas sus facetas, es por supuesto un tema que los involucra, o les «afecta», según los términos de la Convención. Excluirlos derechamente del ámbito legislativo implicaría retroceder de forma brusca en su reconocimiento como sujetos de derechos activos en la toma de decisiones alrededor de sus proyectos de vida. Por otro lado, las limitaciones etarias carecen de sentido, cada vez que se respeten los lineamentos indicados por el Comité sobre la necesidad de hacer análisis caso por caso para determinar la participación de niños, niñas y adolescentes según la evolución de sus facultades y el concepto de autonomía progresiva. Dependiendo de las modalidades en que se legisle sobre ellos, podría potencialmente despertarse la preocupación de los órganos internacionales de supervisión y protección de derechos humanos por la falta de compromiso y de adhesión al corpus iuris de derecho internacional de los derechos humanos vigente en la materia.

\section{Agradecimientos}

Quiero agradecer a Magdalena Abarca Lizana y a Ralph Lorén Eisendecher por haber compartido conmigo sus acertados comentarios sobre el texto.

30. Observación General 12, página 22.

31. Observación General 7, Comité de los Derechos del Niños, 20 de septiembre de 2006, CRC/C/ GC/7/Rev.1, página 76 . 


\section{Referencias}

BARTOS, Ann (2016). «Children and young people's political participation: A critical analysis». En Tracey Skelton (editora), Politics, citizenship and rights. Singapur: Springer.

BURGORGUe-LarSen, Laurence (2013). «Les standards, normes imposées ou consenties?». En Marthe Fatin-Rougue Stefanini (directora), Existe-t-il une exception française en matière de droits fondamentaux? Les Cahiers de l'Institut Louis Favoreu. Marsella: Presses Universitaires d'Aix-Marseille.

$\mathrm{CIDH}$, Comisión Interamericana de Derechos Humanos (2017). Hacia la garantía efectiva de los derechos de niñas, niños y adolescentes: Sistemas nacionales de protección. Washington D.C.: Organización de los Estados Americanos.

Espejo Yaksic, Nicolás y Fabiola Lathrop Gómez (2015). «Identidad de género, relaciones familiares y derechos de niños, niñas y adolescentes: Comentarios al proyecto de ley que reconoce y da protección al derecho a la identidad de género». Revista de Derecho de la Universidad Católica del Norte, 22 (2): 393-418. DOI: 10.4067/So718-97532015000200013.

Holzscheiter, Anna (2010). Children's rights in international politics: The transformative power of discourse. Nueva York: Palgrave Macmillan.

IIN, Instituto Interamericano del Niño, la Niña y Adolescentes (2011). El hecho del dicho. Montevideo.

Keller, Helen y Leena Grover (2012). «General comments of the Human Rights Committee and their legitimacy». En Hellen Keller y Geir Ulfsten (editores), UN human rights treaty bodies: Law and legitimacy (pp. 116-198). Cambridge: Cambridge University Press.

Kilkelly, Ursula (2011). «Using the Convention on the Rights of the Child in law and policy: Two ways to improve compliance». En Antonella Invernizzi y Jane Williams (editores), The human rights of children. From visions to implementation (pp. 179-198). Cardiff: Ashgate.

Lansdown, Gerison (2005). La evolución de las facultades del niño. Florencia: Unicef, Centro de Investigaciones Innocenti.

Meza-Lopehandía, Matías (2018). «Proyecto de ley de identidad de género: Elementos para la discusión legislativa (tercer trámite)». Informe de Asesoría Técnica Parlamentaria, SSP80576. Santiago: Biblioteca del Congreso Nacional.

Mundaca Rojas, Rodrigo y Claudio Flores ZúñIga (2014). «Derechos del niño, participación infantil y formación ciudadana desde espacios educativos no formales: La experiencia del Consejo Consultivo de Niños, Niñas y Adolescentes de la comuna de Coquimbo, Chile». Temas de Educación, 20 (1): 123-141. Disponible en http://bit.ly/2Plexot. 
NACIONES UnidAs (1997). Manual on human rights reporting under six major international human rights instruments. Ginebra.

SERrano, Sandra (2013). Obligaciones del Estado frente a los derechos humanos y sus principios rectores: Una relación para la interpretación y la aplicación de los derechos. Ciudad de México: UNAM.

UNICEF (2007). Implementation handbook for the Convention on the Rights of the Child. Ginebra: United Nations Publications.

\section{Sobre la autora}

Lavinia Francesconi es licenciada en Derecho. Diplomado de postítulo interdisciplinario en Derecho Internacional de los Derechos Humanos por la Facultad de Derecho de la Universidad de Chile. Licenciada en Derecho cum laude por la Facultad de Derecho de la Universidad de Bolonia, Italia. Estudiante en el LL.M. Public International Law de la Facultad de Derecho de la Universidad de Leiden, Países Bajos. 
El Anuario de Derechos Humanos es una publicación anual de referencia y consulta en derechos humanos y materias afines, que busca ser un espacio de discusión, difusión y conocimiento de los temas centrales sobre derechos humanos en sus contextos nacional e internacional, poniendo a la disposición del público de manera gratuita los distintos desarrollos doctrinales, jurisprudenciales y legislativos ocurridos en este campo dentro del período anual cubierto por cada edición.

\author{
DIRECTORA RESPONSABLE \\ Nancy Yáñez Fuenzalida \\ EDITOR DE CONTENIDOS \\ Salvador Millaleo \\ SITIO WEB \\ anuariocdh.uchile.cl \\ CORREO ELECTRÓNICO \\ anuario-cdh@derecho.uchile.cl
}

LICENCIA DE ESTE ARTÍ́CULO

Creative Commons Atribución Compartir Igual 4.0 Internacional

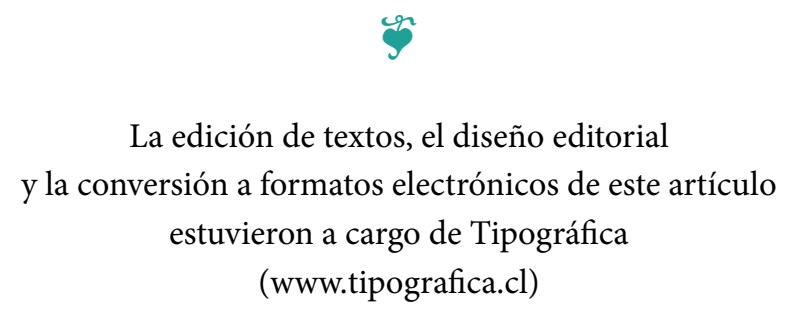

